\title{
(2) OPEN ACCESS \\ Impact of COVID-19 restrictions on diabetes health checks and prescribing for people with type 2 diabetes: a UK-wide cohort study involving 618161 people in primary care
}

\author{
Matthew J Carr (D) , 1,2 Alison K Wright (D) , 1,3 Lalantha Leelarathna (D) , 3 \\ Hood Thabit (D) , ${ }^{3,4}$ Nicola Milne, ${ }^{4}$ Naresh Kanumilli, ${ }^{4}$ \\ Darren M Ashcroft (D) , ${ }^{1,2}$ Martin K Rutter (1) ${ }^{3,4}$
}

- Additional supplemental material is published online only. To view, please visit the journal online (http://dx.doi. org/10.1136/bmjgs-2021013613).

For numbered affiliations see end of article.

\section{Correspondence to}

Dr Matthew I Carr, Centre for Pharmacoepidemiology and Drug Safety, Division of Pharmacy and Optometry, The University of Manchester, Manchester M13 9PL, UK; matthew.carr@manchester. ac.uk

MJC and AKW are joint first authors.

DMA and MKR are joint senior authors.

Received 29 April 2021 Accepted 25 August 2021

A Check for updates

(c) Author(s) (or their employer(s)) 2021. Re-use permitted under CC BY. Published by BMJ.

To cite: Carr MJ, Wright AK, Leelarathna L, et al. BMJ Qual Saf Epub ahead of print: [please include Day Month Year]. doi:10.1136/ bmjqs-2021-013613

\begin{abstract}
Objective To compare rates of performing National Institute for Health and Care Excellence-recommended health checks and prescribing in people with type 2 diabetes (T2D), before and after the first COVID-19 peak in March 2020, and to assess whether trends varied by age, sex, ethnicity and deprivation.

Methods We studied 618161 people with T2D

followed between March and December 2020 from 1744 UK general practices registered with the Clinical Practice Research Datalink. We focused on six health checks: haemoglobin A1c, serum creatinine, cholesterol, urinary albumin excretion, blood pressure and body mass index assessment. Regression models compared observed rates in April 2020 and between March and December 2020 with trend-adjusted expected rates derived from 10-year historical data.

Results In April 2020, in English practices, rates of performing health checks were reduced by $76 \%-88 \%$ when compared with 10 -year historical trends, with older people from deprived areas experiencing the greatest reductions. Between May and December 2020, the reduced rates recovered gradually but overall remained $28 \%-47 \%$ lower, with similar findings in other UK nations. Extrapolated to the UK population, there were 7.4 million fewer care processes undertaken March-December 2020. In England, rates for new medication fell during April with reductions varying from $10 \%$ (95\% Cl: $4 \%$ to $16 \%$ ) for antiplatelet agents to $60 \%$ (95\% Cl: $58 \%$ to $62 \%$ ) for antidiabetic medications. Overall, between March and December 2020, the rate of prescribing new diabetes medications fell by $19 \%$ ( $95 \%$ Cl: $15 \%$ to $22 \%$ ) and new antihypertensive medication prescribing fell by $22 \%$ (95\% Cl: $18 \%$ to $26 \%$ ), but prescribing of new lipid-lowering or antiplatelet therapy was unchanged. Similar trends were observed across the UK, except for a reduction in new lipid-lowering therapy prescribing in the other UK nations (reduction: 16\% (95\% Cl: 10\% to $21 \%)$ ). Extrapolated to the UK population, between March and December 2020, there were 31800 fewer
\end{abstract}

people with T2D prescribed a new type of diabetes medication and $\sim 14600$ fewer prescribed a new type of antihypertensive medication.

Conclusions Over the coming months, healthcare services will need to manage this backlog of testing and prescribing. We recommend effective communications to ensure patient engagement with diabetes services, monitoring and opportunities for prescribing, and when appropriate use of home monitoring, remote consultations and other innovations in care.

\section{INTRODUCTION}

In 2008, the National Institute for Health and Care Excellence (NICE), serving the National Health Service (NHS) in England and Wales, recommended nine essential 'health checks' or so-called 'care processes' that define high-quality diabetes care. ${ }^{1}$ NICE recommended that people with diabetes should have at least annual checks of weight, blood pressure, smoking status, haemoglobin A1c (HbA1c), cholesterol, creatinine, urinary albumin, retinopathy and feet. NICE guidelines apply in England, Wales and Northern Ireland. In Scotland, diabetes guidelines recommend a similar system of health checks, ${ }^{2}$ and these are accepted measures of diabetes care quality worldwide. ${ }^{3-5}$ Since 2008 , these health checks have been incorporated in National Diabetes Audits and have also been used effectively in the Quality Outcomes Framework (QOF) with increasing yearon-year rates of performing these checks, except for albuminuria testing. ${ }^{6-8}$ The 
QOF is a voluntary annual reward and incentive programme for all General Practitioners (GPs) in England and is designed to measure achievements and to reward good practice.

The COVID-19 pandemic has had major health and economic effects across the world. As of 12 August 2021, there have been more than 131000 COVID19-related deaths in the UK, with disproportionate impacts in people with diabetes; in the early phase of the pandemic, nearly one-third of all COVID-19related deaths occurred in people with diabetes. ${ }^{9-11}$ The impact on the NHS, and in particular on diabetes services, has been enormous, with the suspension of much routine care. As the COVID-19 pandemic continues, there is an urgent need to minimise the harm done through reduction of routine services and to prioritise care and resources to areas of greatest need. The management of type 2 diabetes (T2D) occurs almost exclusively in primary care. ${ }^{12}$ Therefore, lower general practice attendance due to COVID-19 would likely restrict the ability to perform these essential health checks. Consequently, this could have adverse effects on patient safety and increase the risk of developing long-term diabetes-related complications.

We have recently shown the indirect consequences of the COVID-19 pandemic on diagnosis rates, HbA1c monitoring and mortality in T2D. ${ }^{13}$ However, there are limited data on the impact of the COVID-19 pandemic on diabetes health checks and prescribing in primary care. Therefore, we used a large primary care longitudinal dataset, broadly representative of the UK population, to compare the frequency of health checks and prescribing in people with T2D, before and after the first nationwide COVID-19 lockdown in March 2020. In UK-wide data, we compared observed and predicted rates using data covering 10 years prior to the pandemic. Since older people, minority ethnic groups and more socially disadvantaged people have been disproportionally affected by COVID-19 infections, and since the same groups may be more adversely impacted by changes in healthcare delivery imposed by COVID-19, we aimed to study variation in outcomes by age, sex, ethnicity and deprivation level.

\section{METHODS}

\section{Data sources}

We conducted a retrospective cohort study using primary care electronic health records obtained from the Clinical Practice Research Datalink (CPRD) Aurum and GOLD databases. ${ }^{13} 14$ The CPRD contains anonymised consultation records and includes patient demographic information, symptoms, diagnoses, medication prescriptions and date of death. In CPRD Aurum, there were 1370 contributing general practices (15\% of all UK general practices): $99 \%$ in England and 1\% in Northern Ireland. ${ }^{15}$ Due to English general practices transitioning from the Vision GP system to the EMIS system, the number of English practices contributing to CPRD GOLD decreased over time. CPRD GOLD was composed of 394 contributing practices (4\% of UK general practices) of which 14\% were in England, 10\% in Northern Ireland, 50\% in Scotland and $26 \%$ in Wales. ${ }^{15}$ We also examined practice-level Index of Multiple Deprivation (IMD) quintiles, ${ }^{14}$ a measure representing an area's relative level of deprivation, ranked within each UK nation.

Our primary analysis was conducted using Aurum data from English practices (covering 99\% of contributing practices in the database). Analyses were replicated in GOLD providing information on practices in Northern Ireland, Scotland and Wales.

\section{Definitions, measurements and clinical coding}

To enable comparisons of rates before and after the start of the COVID-19 outbreak, we included patient records from January 2010 to establish longterm trends and patterns of seasonality. We focused primarily on reporting observed versus expected rates from 1 March 2020 to 10 December 2020. We also chose to study rates of health checks and prescribing in April 2020 since this was the first full month following the national lockdown. For the diabetes monitoring component of the study, we restricted our investigation to the following six care processes because there was a high level of confidence that they had or had not been performed based on the available primary care records: HbA1c, serum creatinine, cholesterol, urinary albumin excretion, blood pressure, body mass index (BMI) measurement. It seemed possible that smoking status had been assessed and foot checks performed but not recorded in the practice records and therefore we did not report these outcomes. Several novel models of diabetes-related foot care have evolved during the pandemic ${ }^{16}{ }^{17}$ and therefore we felt that primary care data alone might not adequately capture foot assessment activity. Eye screening for retinopathy is performed by optometrists that are not linked to GP practices and the results are not routinely captured in the primary care record.

For the medication prescribing component of the study, we focused on medications commonly prescribed to patients with a diagnosis of T2D: antidiabetics, antihypertensives, lipid-lowering drugs and antiplatelet agents. To compare prescribing behaviours before and after the start of the pandemic, we applied two distinct definitions: first, we assessed the prescribing of new medication within a 3-year window of a first diagnosis of T2D where new prescriptions were identified among those eligible to receive new treatment, that is, people within 3 years of diagnosis not currently or historically prescribed the medication of interest. Second, we assessed the overall prescribing rate (new and repeat) among patients with a prior diagnosis.

We also compared the incidence and event rates for eight separate strata by combining attributes of the study cohort via the dichotomisation of sex, age (less 
than 65 years vs greater than or equal to 65 years), and IMD (quintiles 4 and 5 (most deprived) vs quintiles 1 , 2 and 3) as shown in online supplemental table 1.

Care processes were identified using Read/ SNOMED/EMIS codes used in CPRD GOLD and Aurum. Medication prescribing events were identified using CPRD product codes linked to codes from the dictionary of medicines and devices. All medical and drug code lists used in the analyses are available as online supplemental appendices.

Ethnicity was classified from primary care and Hospital Episode Statistics (HES) records as white/ Asian/black/mixed/other or white/other dependent on sample size. For patients with multiple records and conflicts, we defined ethnicity as (a) the category with most corresponding records; (b) according to the last available record when record numbers were tied across two or more categories; and (c) by randomly selecting between candidate ethnicities when record numbers were tied across two or more categories and multiple categories were entered in the last available record.

All code lists and medication lists were verified by two senior clinical academics (a diabetologist: MKR, and a senior academic pharmacist: DMA).

\section{Study design}

For each patient, we defined a 'period of eligibility' for study inclusion which commenced on the latest of: the study start date (1 January 2010); the patient's most recent registration with their practice; the date on which data from the practice were deemed to be 'up-to-standard' by the CPRD; the patient's first diagnosis of T2D. A patient's period of eligibility ended on the earliest of: registration termination; the end of data collection from their practice; death. We also applied a 'look-back' period during which a patient was required to have been registered for at least a year prior to their diagnosis of T2D. The denominator for the incidence and event rates was the aggregate personmonths at risk for the whole eligible study cohort. With our open-cohort design, patients could enter or exit the cohort at any time during the study period depending on their date of registration, diagnosis and migration from their practice. As such, a given patient in a specific month could contribute a full or partial month of follow-up to the person-time denominator.

\section{Statistical analysis}

The data were structured in a time-series format with event counts and 'person-months at risk' aggregated (by year and month) with stratification by sex, age group, ethnicity, deprivation quintile and region (or nation in $G O L D)$. Mean-dispersion negative binomial regression models were used to estimate expected monthly event counts from March 2020 onward based on antecedent trends since 2010. The natural logarithm of the denominator (person-months at risk) was used as an offset in each regression model. To account for possible seasonality and long-term linear trends, calendar month was fitted as a categorical variable and time as a continuous variable with the number of months since the start of the study serving as the unit of measurement. For each month studied, observed and expected event counts were converted to rates using the observed person-month denominator. The monthly expected rates, and their 95\% CIs, were plotted against the observed rates. As they share a common denominator, differences between expected and observed monthly rates are expressed as a percentage 'rate reduction (or increase)'.

Although it was not possible to directly estimate the number of patients who missed out on a given care process, we estimated the shortfall in the number of care processes undertaken between March and December 2020 and the number of patients prescribed new medications.

All data processing and statistical analyses were conducted using Stata V.16 (StataCorp, College Station, Texas, USA). We followed REporting of studies Conducted using Observational Routinelycollected health Data (RECORD) guidance. ${ }^{18}$

\section{RESULTS}

Study cohort

Our focus was on the impact of the COVID-19 pandemic between March and December 2020. Using the inclusion criteria described in the Study design section, a mixed cohort was used consisting of those patients from the study population whose period of eligibility began before 1 March 2020 and those who became eligible for inclusion between 1 March 2020 and 10 December 2020 (online supplemental figures 1 and 2). The study population consisted of 965964 patients with a diagnosis of T2D: 824698 patients from 1470 general practices in England, with a further 141266 patients from 361 practices across Northern Ireland (16408 patients in 40 practices), Scotland (69935 patients in 208 practices), and Wales (54923 patients in 113 practices). From the study population, 934214 patients (from 1828 UK general practices) contributed to the estimation of the expected rates in the pre-COVID-19 period between January 2010 and February 2020. The study cohort that contributed to the comparisons of observed and expected rates between March and December 2020 comprised 618161 people with T2D from 1744 UK general practices. The median (IQR) age was 68 (58-77) years, $44 \%$ were female and $25 \%$ lived in an area that was in the most deprived quintile compared with the rest of the UK.

Ethnicity data were available in 699572 (85\%) of those with T2D in England and 64457 (46\%) of those in Northern Ireland, Scotland and Wales with the following breakdown: England (white 80\%, Asian $13 \%$, black $6 \%$, mixed $1 \%$, other $0.4 \%$ ); other UK 


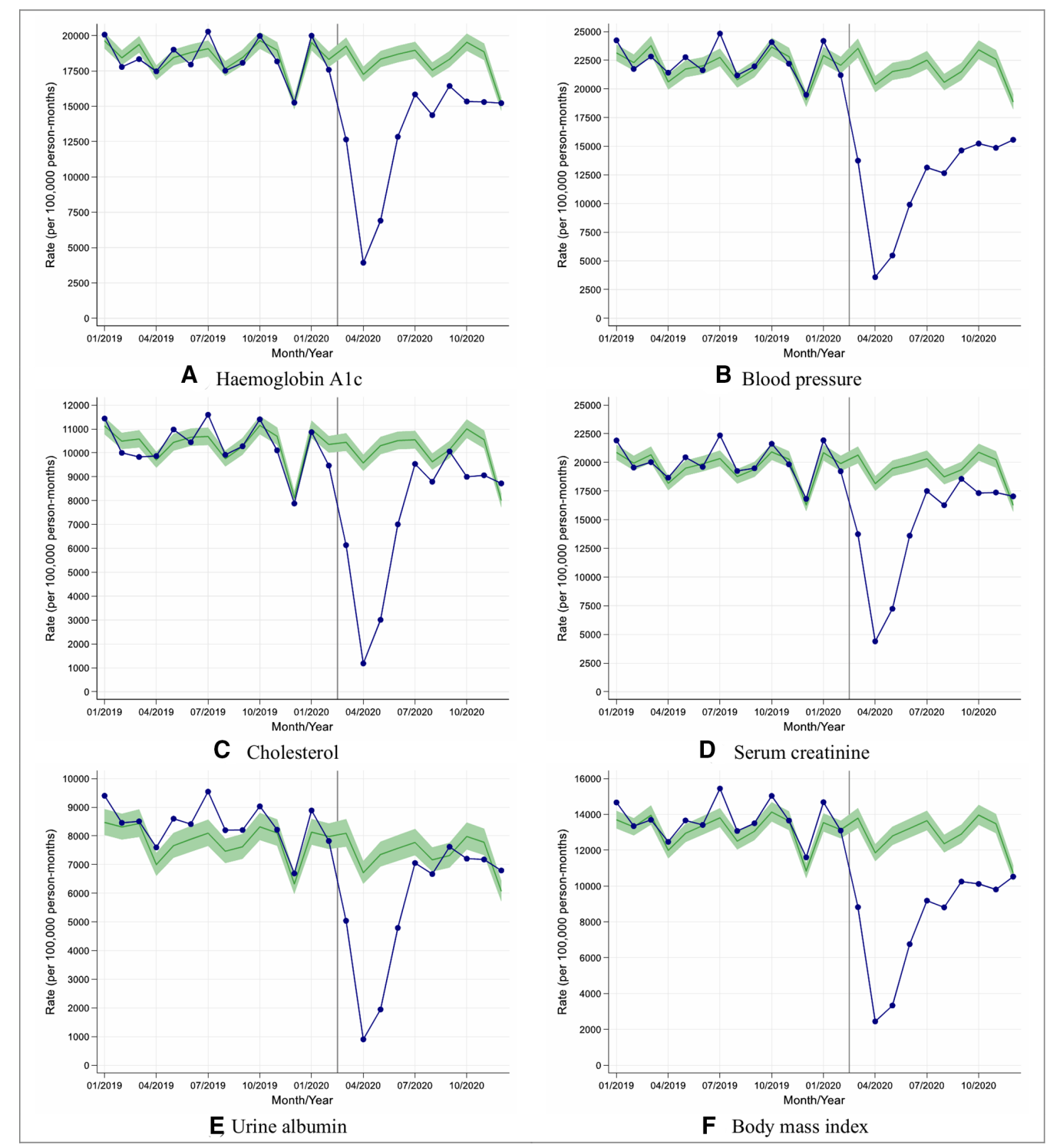

Figure 1 Observed and expected care process rates in people with type 2 diabetes during 2019 and 2020, in England. (A-F) Blue lines indicate observed monthly rates (years 2019 and 2020), and green-shaded regions indicate expected rates with 95\% Cls based on 10-year historical trends from January 2010; the vertical line at 1 March 2020 separates the rates in primary care before and after the start of the COVID-19 pandemic, and x-axis markers indicate mid-months. Created by the authors.

nations (white 96\%, Asian 3\%, black 0.5\%, mixed $0.2 \%$, other $0.2 \%$ ).

\section{Impacts of COVID-19 on care processes in T2D}

In April 2020, in English primary care, the rate of performing health checks was reduced by 76\%-88\% when compared with 10-year historical trends (figure 1), with similar reductions (74\%-88\%) in other UK nations (online supplemental figure 3).

Although reductions in rates of performing health checks were similar by age, sex and socioeconomic group, older people from deprived areas tended to have the greatest reductions in rates due to having higher background testing rates (England, figure 2; other UK nations, online supplemental figure 4).

Between May and December 2020, the reduced rates of performing health checks recovered gradually though rates remained well below expected especially for blood pressure and BMI monitoring (England, figure 1; other UK nations, online supplemental figure $3)$.

Overall in English practices, between 1 March and 10 December 2020, the rate of performing each of the health checks was reduced by between $28 \%$ and $47 \%$ compared with historical trends (table 1); the most affected health check being blood pressure testing (rate reduction (95\% CI): $47 \%$ (45\% to $49 \%)$ ) and the least affected being urine albumin monitoring (reduction $(95 \% \mathrm{CI}): 28 \%(23 \%$ to $32 \%))$. Similar trends were observed in other UK nations with rate reductions varying between $37 \%$ and $51 \%$ across the health checks (online supplemental table 2); blood pressure monitoring being most significantly reduced (rate reduction (95\% CI): 51\% (49\% to 53\%)). 


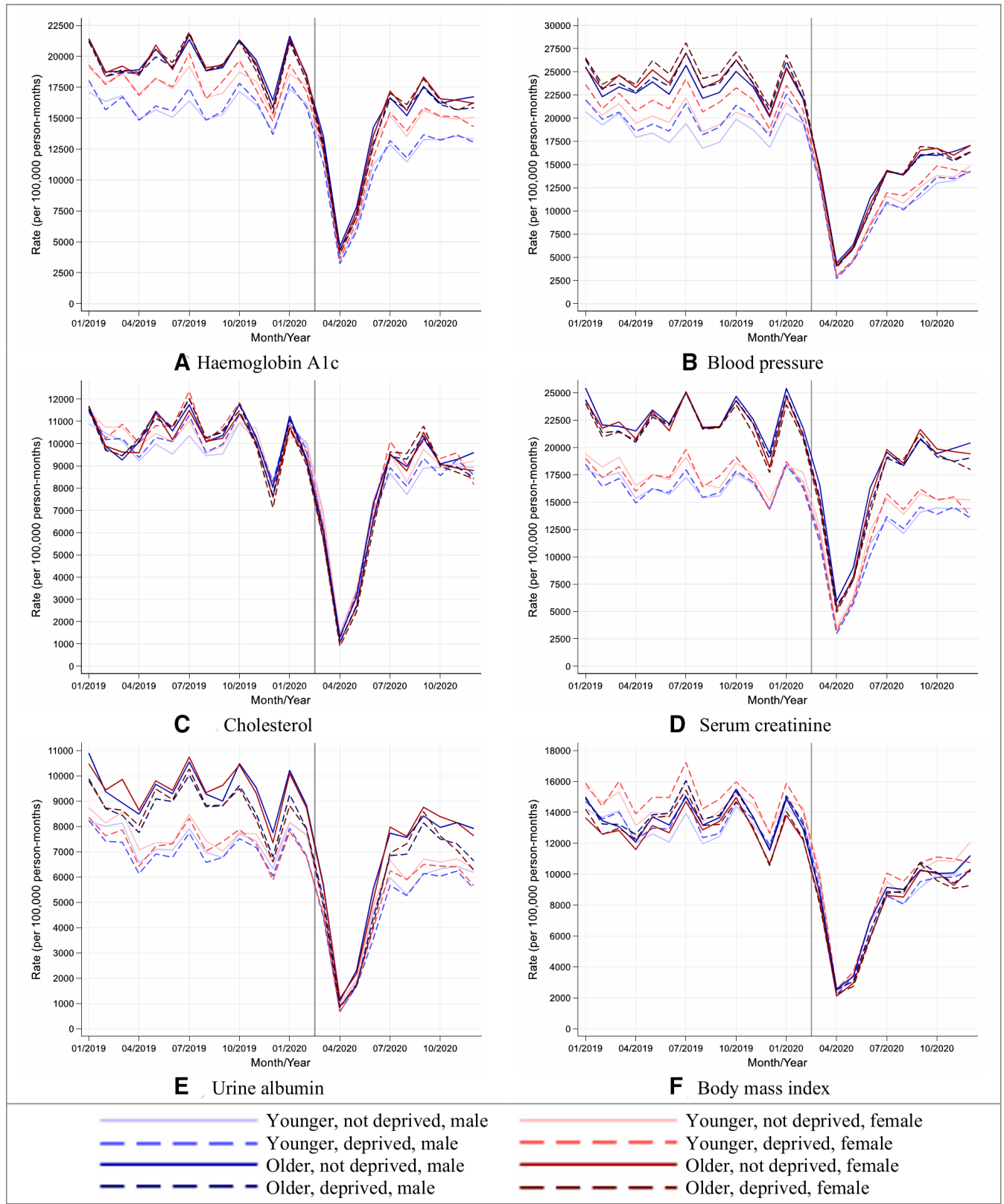

Figure 2 Stratified care process rates in people with type 2 diabetes during 2019 and 2020, in England. (A-F) Lines indicate observed monthly rates (years 2019 and 2020); the vertical line at 1 March 2020 separates the rates in primary care before and after the start of the COVID-19 pandemic, and $x$-axis markers indicate mid-months. Created by the authors.

Similar patterns were observed in ethnicity-stratified analysis in England (online supplemental figure 8) and in other UK nations (online supplemental figure 9).

Extrapolated to the UK population, there were $\sim 7.4$ million fewer care processes undertaken between March and December 2020: 2.3 million of these being blood pressure checks and $\sim 1.2$ million being HbA1c checks (online supplemental tables 3 and 4).

\section{Impacts of COVID-19 on diabetes-related prescribing}

We assessed changes in rates of prescribing of new diabetes medication along with new antihypertensive, lipid-lowering and antiplatelet medications in people with T2D. In England, prescribing of new medication fell during April with rate reductions varying from $10 \%$ (95\% CI: $4 \%$ to $16 \%$ ) for antiplatelet agents to $60 \%$ (95\% CI: $58 \%$ to $62 \%$ ) for antidiabetic medications (figure 3). Similar patterns were observed in other UK nations with rate reductions varying between 26\% (95\% CI: $15 \%$ to 34\%) for antiplatelet agents and $64 \%$ (95\% CI: $61 \%$ to $66 \%$ ) for lipid-lowering therapy (online supplemental figure 5).

In contrast to the data on rates of performing care processes, the largest reductions in rates of prescribing new diabetes medication and new lipid-lowering medication in England were seen in younger individuals from deprived and non-deprived backgrounds (online supplemental figure 6). 


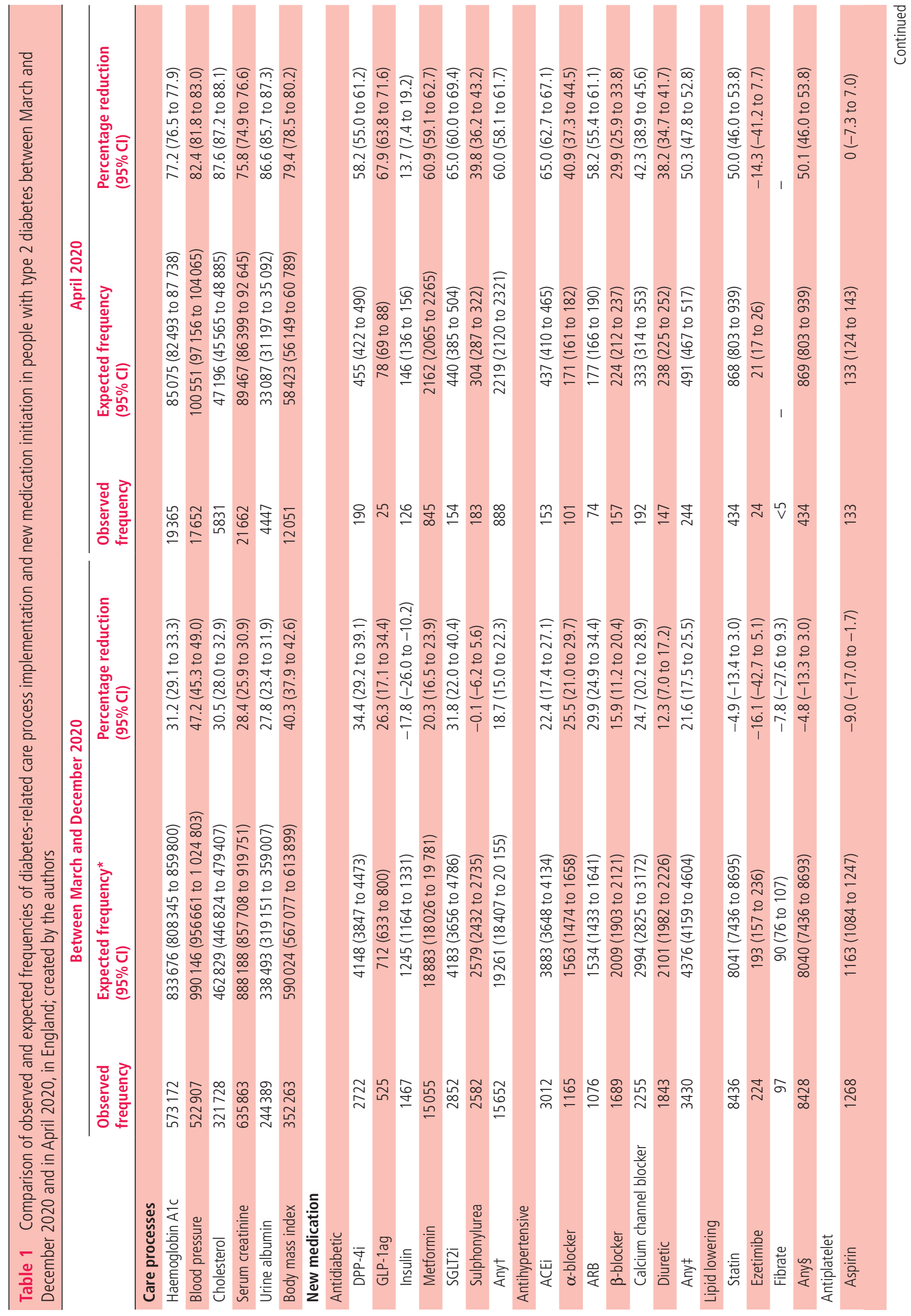




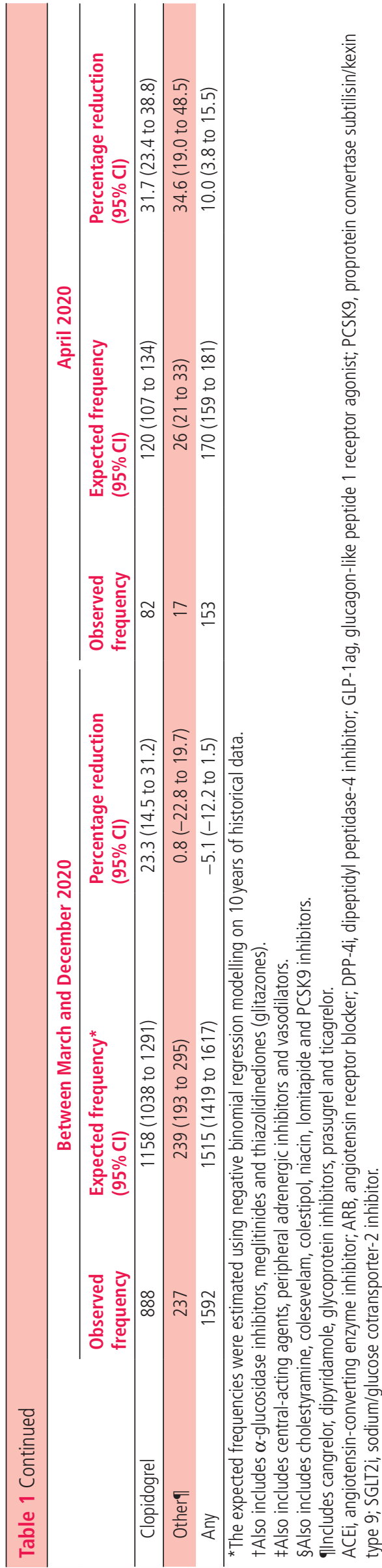

Overall in English practices, between 1 March and 10 December 2020, the overall rate of prescribing new diabetes medications was reduced by 19\% (95\% CI: $15 \%$ to $22 \%$ ) when compared with historical trends (table 1); the most affected medication being dipeptidyl peptidase-4 inhibitors (reduction (95\% CI): 34\% $(29 \%$ to $39 \%))$ and the least affected being insulin which was initiated more frequently during this period compared with historical trends (increase $(95 \% \mathrm{CI})$ : $18 \%(10 \%$ to $26 \%))$.

Similarly, the prescribing of new antihypertensive medication was reduced by $22 \%$ (95\% CI: $18 \%$ to $25 \%$ ) during this period whereas there was no significant change in the prescribing of new lipid-lowering or new antiplatelet therapy (table 1).

Between 1 March and 10 December 2020, similar reductions in the trends for prescribing of new medication were observed in other UK nations except that there was a significant reduction in new lipid-lowering therapy prescribing (reduction $(95 \% \mathrm{CI}): 15 \%(10 \%$ to $21 \%$ ); online supplemental table 2).

Similar patterns of prescribing were observed in ethnicity-stratified analysis in England (online supplemental figure 10); data being too sparse to provide conclusive results in other UK nations.

Extrapolated to the UK population, between March and December 2020, there were $\sim 31800$ fewer people with T2D prescribed a new type of diabetes medication including $\sim 17100$ without a prior prescription of any first antidiabetic. Similarly, there were $\sim 14600$ fewer people prescribed a new type of antihypertensive medication including $\sim 4500$ without a prior prescription of any first antihypertensive (online supplemental tables 3 and 4).

When considering both new prescribing and repeat prescribing combined, there was no significant change in the prescribing of antidiabetic, antihypertensive, lipid-lowering or antiplatelet therapies between March 2020 and December 2020 (England, figure 4; other UK nations, online supplemental figure 7).

\section{DISCUSSION}

We used primary care electronic health records from more than 600000 people with T2D in the UK, and 10-year historical data, to show that during the 9 months following the first nationwide 'lockdown', the indirect consequences of the COVID-19 pandemic were associated with clinically significant changes in care quality and prescribing that could adversely impact patient safety. When compared with historical trends, we showed that: (1) there were $28 \%-47 \%$ reductions in rates of performing a range of health checks including a near halving of blood pressure testing rates; (2) older people with T2D and those from more deprived backgrounds experienced the greatest reduction in health checks; (3) overall rates of prescribing new diabetes and antihypertensive medication were reduced by $19 \%-22 \%$; and (4) reassuringly, 


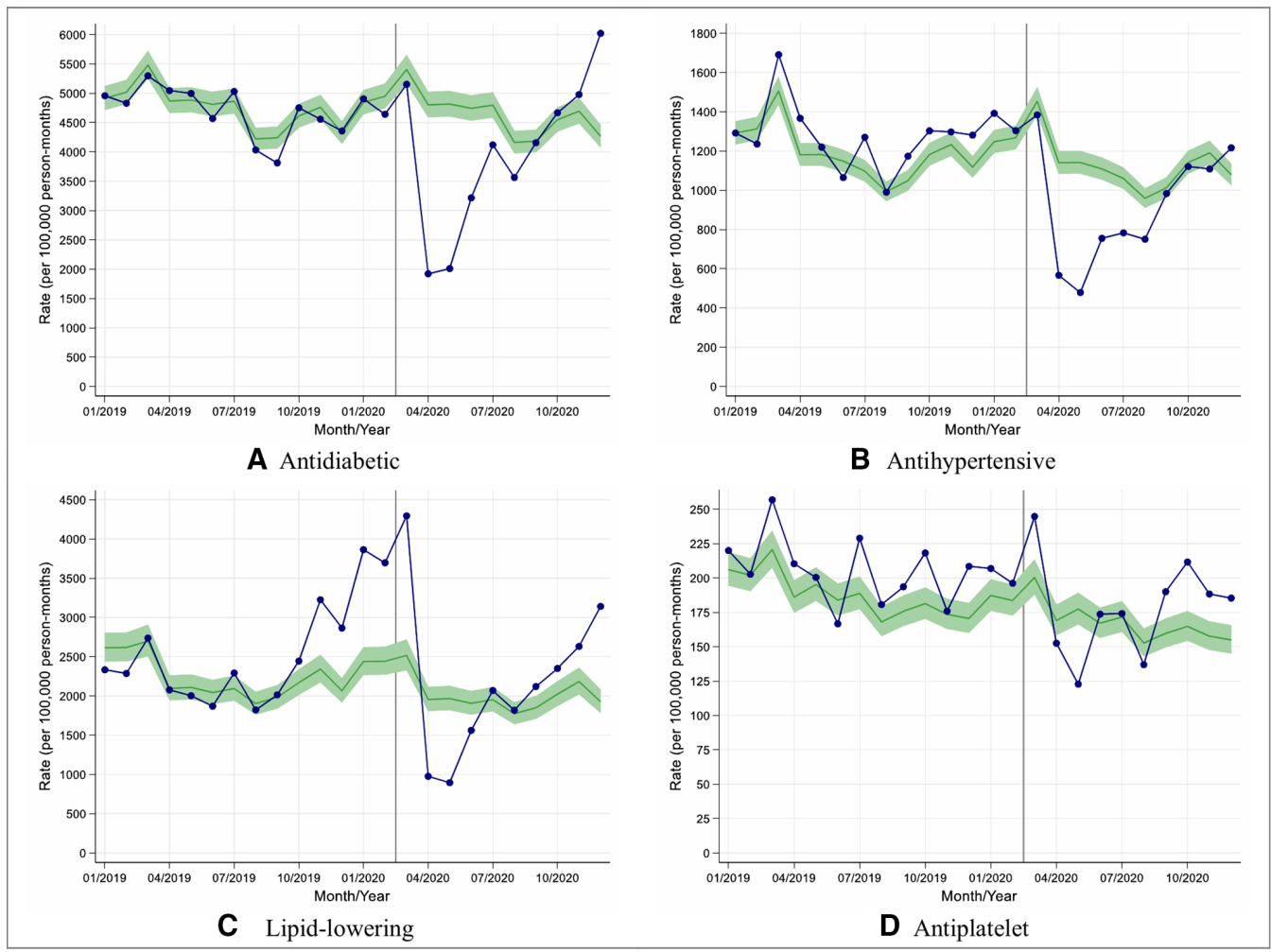

Figure 3 Observed and expected rates of new medication initiation in people with type 2 diabetes during 2019 and 2020, in England. (A-D) Blue lines indicate observed monthly rates (years 2019 and 2020), and green-shaded regions indicate expected rates with 95\% Cls based on 10-year historical trends from January 2010; the vertical line at 1 March 2020 separates the rates in primary care before and after the start of the COVID-19 pandemic, and x-axis markers indicate mid-months. Created by the authors.

when considering rates of new and repeat medication prescribing combined, there were no significant differences.

To provide some context for our health check data, National Diabetes Audit data indicate that during the 15 months prior to the national lockdown in March 2020, most people with T2D in England had the six health checks performed: haemoglobin A1c (94\% of people), serum creatinine (92\%), cholesterol (91\%), urinary albumin excretion (69\%), blood pressure $(95 \%)$ and BMI $(88 \%){ }^{7}$

There are limited data on the impact of the COVID-19 pandemic on rates of performing health checks and prescribing in people with T2D diabetes. An earlier UK-wide study using primary care data from people with T2D showed a 31\% reduction in HbA1c testing, a 20\% reduction in new metformin prescribing and $5 \%$ reduction in new insulin prescribing between March and December 2020 compared with historical trends. ${ }^{13}$ Here we extend these observations by assessing the impact of the COVID-19 pandemic on a much wider range of health checks and a wider range of diabetes-related medication including agents that reduce cardiovascular risk.

Our findings have important clinical implications for diabetes care quality and safety. In early March 2020, GPs were advised to minimise the number of face-toface contacts they had with their patients. ${ }^{19-22}$ Our data suggest that this reduction of clinical services has contributed to major reductions in the monitoring of $\mathrm{T} 2 \mathrm{D}$ and the prescribing of new medication, particularly for hypertension. T2D is a progressive condition and therefore without intervention, levels of glucose and associated cardiovascular disease (CVD) risk factors such as blood pressure tend to increase over time. It is concerning that we observed a $22 \%$ reduction in new antihypertensive medication prescribing between March and December 2020, perhaps caused by less frequent monitoring and restricted face-to-face clinical contacts. Anecdotally, we understand that some practices were reluctant to prescribe ACE inhibitors as they would not be able to routinely check renal bloods after initiation. There are already concerns about clinical inertia in diabetes management in the $\mathrm{UK},{ }^{23}$ and therefore any further reduction in monitoring and related prescribing could increase the risk of mortality and long-term complications.

Our data indicate that reductions in prescribing relate to new prescriptions but not repeat prescriptions. Robust systems for repeat prescribing in the UK appear to have helped minimise the harm done through reductions in face-to-face consultations during the pandemic.

The effect of COVID-19 lockdown restrictions on metabolic control in people with diabetes appears heterogeneous. Some studies in insulin-treated people 


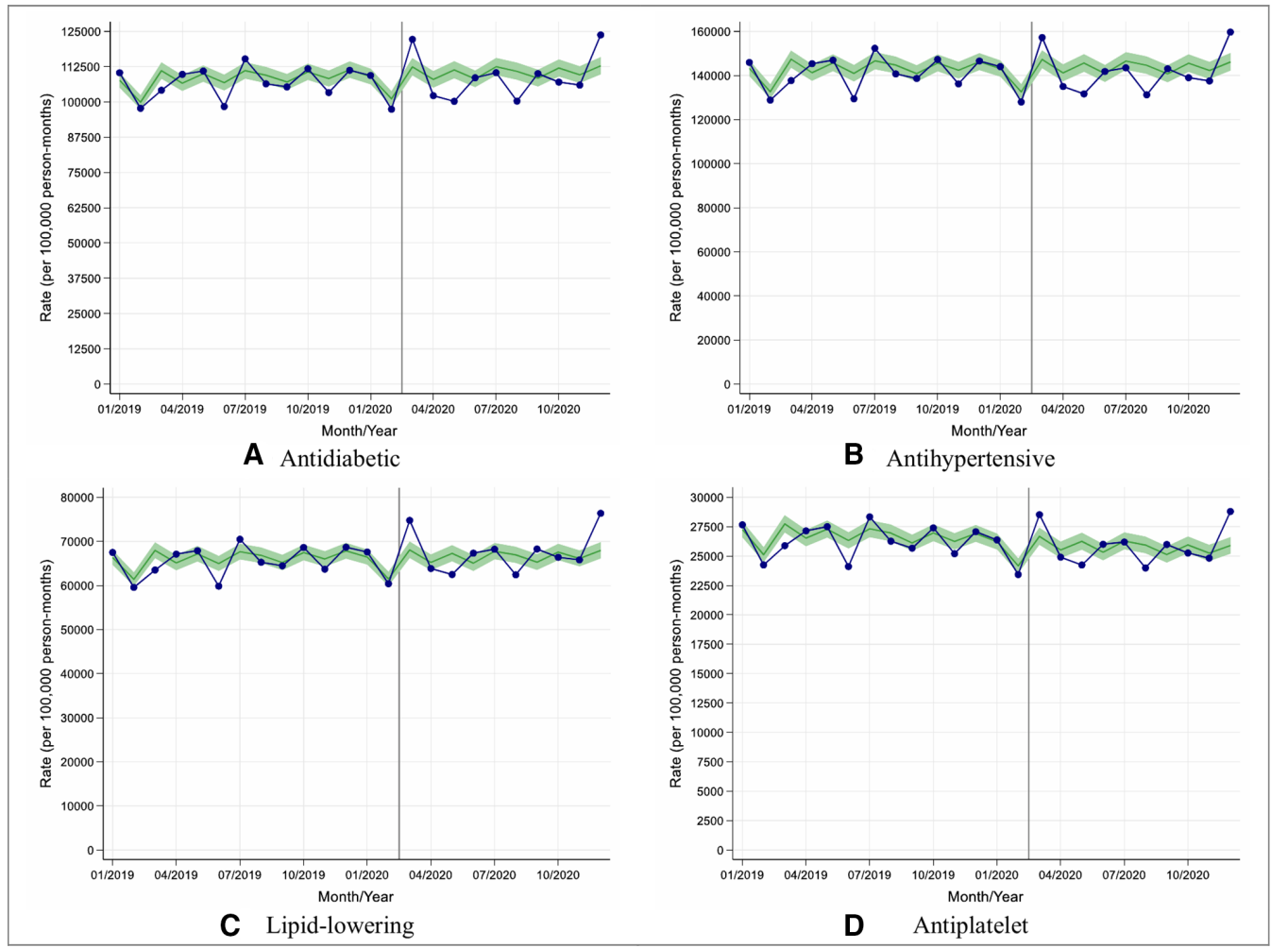

Figure 4 Observed and expected new and repeat medication prescribing rates in people with type 2 diabetes during 2019 and 2020, in England. (A-D) Blue lines indicate observed monthly rates (years 2019 and 2020), and green-shaded regions indicate expected rates with $95 \%$ Cls based on 10 year historical trends from January 2010; the vertical line at 1 March 2020 separates the rates in primary care before and after the start of the COVID-19 pandemic, and $\mathrm{x}$-axis markers indicate mid-months. Created by the authors.

have suggested improvements in glucose control perhaps brought about through more regular mealtimes, increased consumption of homemade foods, decreased workloads, less exercise and more time for self-care. ${ }^{24-26}$

However, in many people with T2D, there is evidence that national lockdowns have had detrimental effects on CVD risk, which could be exacerbated by the reduced monitoring and prescribing observed in our study. In surveys of UK adults conducted during the first lockdown (April--May 2020), participants reported adverse changes in several behaviours that promote weight gain (adverse changes in diet, physical activity, alcohol consumption, mental health and sleep quality). ${ }^{27}{ }^{28}$ Some $^{29-31}$ but not all studies ${ }^{32} 33$ in people with T2D have shown worsening of glycaemic control in relation to COVID-19 lockdown which could explain the increased rates of insulin prescribing we observed between March and December 2020.

As engagement with health services increases over the coming months, we predict marked increases in the need for monitoring and prescribing of new medication in people with T2D. Healthcare services will need to manage this backlog of testing and prescribing, and the anticipated greater deterioration of $\mathrm{HbA} 1 \mathrm{c}$ and other CVD risk factors such as blood pressure levels. Older people from deprived backgrounds appear to be most adversely affected by reduced monitoring and therefore these individuals may be particular groups to target for early intervention. During this pandemic and its associated lockdowns, effective communications should ensure that patients remain engaged with diabetes services, monitoring and opportunities for prescribing, ${ }^{34}$ and make use of home monitoring of blood pressure, weight and HbA1c (when available), and remote consultations. ${ }^{35-37}$ Investment in such technology and devices would be expected to yield important health benefits.

The COVID-19 pandemic provides us with a unique opportunity to improve the current care model by providing greater investment in patient education, devices and technology and greater use of remote consultations to deliver the high standards of care that people with diabetes should expect to receive.

Although our data relate directly to people with T2D, they have relevance to other medical conditions. We, ${ }^{38} 39$ and others, ${ }^{40}$ have shown substantial reductions in primary care contacts for a wide range of physical and mental health conditions following COVID-19 restrictions. Maintaining healthcare access for all should be a key priority in public health planning.

Our study had several strengths: this is the first UK-wide study reporting the indirect impact of the COVID-19 pandemic on health checks and prescribing in people with T2D. Our findings in English practices 
were replicated in other parts of the UK and are likely to be representative of the UK in general. Our study has some limitations: first, we did not report data on retinopathy, smoking and foot checks (the remaining three of the nine health checks recommended by NICE). Retinopathy screening is performed in the community and therefore these data are not available in primary care records. While assessments of smoking status and foot checks are performed in primary care, we were less confident in defining whether or not these checks had been performed based on the available data. Second, we do not present data on type 1 diabetes as the majority of care for these individuals is delivered in secondary care centres. Third, ethnicity coding is not adequately captured in primary care and therefore we had limited ability to explore ethnicity-related variation in outcomes. Fourth, we did not assess risk factor levels because our focus was on processes of care and prescribing. Fifth, our data would not capture assessments of weight and blood pressure assessed by patients in their homes. Results of home blood pressure recordings may have had an influence on prescribing between March and December 2020 because the reduction in prescribing of new antihypertensive agents $(\sim 13 \%)$ was less than the reduction in blood pressure monitoring performed in primary care $(\sim 51 \%)$. Finally, although our results and conclusions are relevant to the UK population, generalisability to other healthcare systems may be limited. However, a pan-European survey of diabetes specialist nurses reported that the level of care provided for people with diabetes had been significantly disrupted during the pandemic. ${ }^{34}$

In conclusion, we highlight marked reductions in the rate of health checks and new prescribing in people with T2D as indirect consequences of the COVID-19 pandemic. Over the coming months, healthcare services will need to manage this backlog of testing and prescribing, and the anticipated greater deterioration of HbA1c and other CVD risk factors such as blood pressure levels. Older people from deprived backgrounds with T2D may be specific groups to target for early health checks and intervention.

During the next year, effective communications should ensure that patients remain engaged with diabetes services including opportunities for prescribing and that they make use of home monitoring of blood pressure, weight, foot health and blood glucose when appropriate. Telemedicine, digital services and remote consultations may provide opportunities to engage some groups of people living with diabetes who have been difficult to reach. Healthcare planners should seize opportunities provided by the COVID-19 pandemic to improve models, processes and standards of care for people with diabetes. A positive lasting legacy of COVID-19 might be accelerated innovation in diabetes care and other chronic diseases. ${ }^{35}$
Author affiliations

${ }^{1}$ Centre for Pharmacoepidemiology and Drug Safety, Division of Pharmacy and Optometry, The University of Manchester, Manchester, UK

${ }^{2}$ NIHR Greater Manchester Patient Safety Translational Research Centre, University of Manchester, Manchester, UK

${ }^{3}$ Division of Diabetes, Endocrinology and Gastroenterology, School of Medical Sciences, The University of Manchester, Manchester, UK

${ }^{4}$ Diabetes, Endocrinology and Metabolism Centre, Manchester University NHS Foundation Trust, Manchester, UK

Twitter Matthew J Carr @PharmacyDUPP, @meds_safety, @PSTRC_GM, Alison K Wright@_AlisonWright, Martin K Rutter@- RutterMK

Acknowledgements The study is based on data from the Clinical Practice Research Datalink obtained under license from the UK Medicines and Healthcare products Regulatory Agency. The study and use of CPRD data were approved by the Independent Scientific Advisory Committee for Clinical Practice Research Datalink research (protocol number:

20_182R). The data are provided by patients and collected by the NHS as part of their care and support. The interpretation and conclusions contained in this study are those of the authors alone. We would like to acknowledge all the data providers and general practices that made the anonymised data available for research.

Contributors DMA conceived the original idea. MKR, MJC and AKW helped develop the idea. MJC and AKW performed the analysis and verified the analytical methods. MKR and DMA also reviewed the clinical code sets. NK and NM (primary care clinicians), and LL and HT (secondary care diabetes clinicians) helped interpret the results. MKR wrote the manuscript with input from all authors. All authors critically reviewed and approved the final version. The lead authors (MJC and AKW: the manuscript's guarantors) affirm that the manuscript is an honest, accurate, and transparent account of the study being reported; that no important aspects of the study have been omitted; and that any discrepancies from the study as planned have been explained. The corresponding author had full access to all of the data and the final responsibility to submit for publication.

Funding This work is funded by the National Institute for Health Research (NIHR) Greater Manchester Patient Safety Translational Research Centre (PSTRC-2016-003).

Disclaimer The funding source had no role in the study design, data collection, data analysis, data interpretation or writing of the report. The views expressed are those of the authors and not necessarily those of the NIHR or the Department of Health and Social Care.

Competing interests DMA reports research funding from AbbVie, Almirall, Celgene, Eli Lilly, Novartis, Janssen, UCB and the Leo Foundation outside the submitted work. MKR has received consulting fees and non-promotional lecture fees from Novo Nordisk in relation to cardiovascular disease and diabetes. The company has had no role in influencing the proposed study and is not expected to benefit from this work. Outside the submitted work, MKR reports receiving research funding from Novo Nordisk, consultancy fees from Novo Nordisk and Roche Diabetes Care, and modest owning of shares in GlaxoSmithKline. NM reports honorarium for presentations from Napp Pharmaceuticals, Novo Nordisk, Sanofi, MyLan, Boehringer Ingelheim, Lilly Diabetes, Abbott, Omnia-Med, Takeda UK and AstraZeneca. All other authors declare no competing interests.

Patient consent for publication Not required.

Provenance and peer review Commissioned; internally peer reviewed.

Data availability statement Data may be obtained from a third party and are not publicly available.

Supplemental material This content has been supplied by the author(s). It has not been vetted by BMJ Publishing Group Limited (BMJ) and may not have been peer-reviewed. Any 
opinions or recommendations discussed are solely those of the author(s) and are not endorsed by BMJ. BMJ disclaims all liability and responsibility arising from any reliance placed on the content. Where the content includes any translated material, BMJ does not warrant the accuracy and reliability of the translations (including but not limited to local regulations, clinical guidelines, terminology, drug names and drug dosages), and is not responsible for any error and/or omissions arising from translation and adaptation or otherwise.

Open access This is an open access article distributed in accordance with the Creative Commons Attribution 4.0 Unported (CC BY 4.0) license, which permits others to copy, redistribute, remix, transform and build upon this work for any purpose, provided the original work is properly cited, a link to the licence is given, and indication of whether changes were made. See: https://creativecommons.org/licenses/by/4.0/.

\section{ORCID iDs}

Matthew J Carr http://orcid.org/0000-0001-7336-1606 Alison K Wright http://orcid.org/0000-0002-8418-8332 Lalantha Leelarathna http://orcid.org/0000-0001-9602-1962

Hood Thabit http://orcid.org/0000-0001-6076-6997

Darren M Ashcroft http://orcid.org/0000-0002-2958-915X

Martin K Rutter http://orcid.org/0000-0001-6380-539X

\section{REFERENCES}

1 National Institute for Health and Care Excellence. Type 2 diabetes clinical guidance [CG66], 2008.

2 Scottish Intercollegiate guidelines network (sign) guideline. management of diabetes, 2017. Available: https://www.sign. ac.uk/our-guidelines/management-of-diabetes/ [Accessed 6 Jul 2021].

3 Badawi D, Saleh S, Natafgi N, et al. Quality of type II diabetes care in primary health care centers in Kuwait: employment of a diabetes quality indicator set (DQIS). PLoS One 2015;10:e0132883.

4 Si D, Bailie R, Wang Z, et al. Comparison of diabetes management in five countries for general and Indigenous populations: an Internet-based review. BMC Health Serv Res 2010;10:169.

5 Tanaka H, Tomio J, Sugiyama T, et al. Process quality of diabetes care under favorable access to healthcare: a 2-year longitudinal study using claims data in Japan. BMJ Open Diabetes Res Care 2016;4:e000291.

6 National diabetes audit executive summary 2008-2009, 2010. Available: https://files.digital.nhs.uk/publicationimport/ pub02xxx/pub02577/nati-diab-audi-08-09-exec-summ.pdf [Accessed 12 Aug 2021].

7 NHS Digital.. National diabetes Audit- care processes and treatment targets 2019-20, 2020. Available: https://digital. nhs.uk/data-and-information/publications/statistical/nationaldiabetes-audit/care-processes-and-treatment-targets-2019-20data-release\#summary [Accessed 6 Jul 2021].

8 Kontopantelis E, Reeves D, Valderas JM, et al. Recorded quality of primary care for patients with diabetes in England before and after the introduction of a financial incentive scheme: a longitudinal observational study. BMJ Qual Saf 2013;22:53-64.

9 Raleigh VS. Uk's record on pandemic deaths. BMJ 2020;370:m3348.

10 Holman N, Knighton P, Kar P, et al. Risk factors for COVID19-related mortality in people with type 1 and type 2 diabetes in England: a population-based cohort study. Lancet Diabetes Endocrinol 2020;8:823-33.

11 Barron E, Bakhai C, Kar P, et al. Associations of type 1 and type 2 diabetes with COVID-19-related mortality in England: a whole-population study. Lancet Diabetes Endocrinol 2020;8:813-22.

12 Alshamsan R, Millett C, Majeed A, et al. Has pay for performance improved the management of diabetes in the United Kingdom? Prim Care Diabetes 2010;4:73-8.

13 Carr MJ, Wright AK, Leelarathna L, et al. Impact of COVID-19 on diagnoses, monitoring, and mortality in people with type 2 diabetes in the UK. Lancet Diabetes Endocrinol 2021;9:413-5.

14 Padmanabhan S, Carty L, Cameron E, et al. Approach to record linkage of primary care data from clinical practice research Datalink to other health-related patient data: overview and implications. Eur J Epidemiol 2019;34:91-9.

15 The clinical practice research Datalink. release notes: CPRD aurum, 2021. Available: https://cprd.com/sites/default/files/ 2021-01\%20CPRD\%20Aurum\%20Release\%20Notes.pdf [Accessed 12 Aug 2021].

16 Rastogi A, Hiteshi P, Bhansali A A, et al. Virtual triage and outcomes of diabetic foot complications during Covid-19 pandemic: a retro-prospective, observational cohort study. PLoS One 2021;16:e0251143.

17 Shin L, Bowling FL, Armstrong DG, et al. Saving the diabetic foot during the COVID-19 pandemic: a tale of two cities. Diabetes Care 2020;43:1704-9.

18 Benchimol EI, Smeeth L, Guttmann A, et al. The reporting of studies conducted using observational Routinely-collected health data (record) statement. PLoS Med 2015;12:e1001885.

19 NHS England and NHS Improvement. Remote working in primary care guidance for GP practices during COVID-19 emergency response, 2020. Available: https://www.england. nhs.uk/coronavirus/wp-content/uploads/sites/52/2020/03/ C0165-remote-working-in-primary-care-gp-practices-duringcovid-19-v1.2.pdf [Accessed 6 Jul 2021].

20 Welsh Government. Simple steps to help your GP practice help you, 2020. Available: https://gov.wales/simple-steps-help-yourgp-practice-help-you [Accessed 6 Jul 2021].

21 Joint Scottish Government, BMA, RCGP. Joint letter, 2020. Available: https://www.sehd.scot.nhs.uk/publications/ DC20200313covid.pdf [Accessed 6 Jul 2021].

22 Public Health Agency in Northern Ireland. PHA advice on new coronavirus guidance, 2020. Available: https://www. publichealth.hscni.net/sites/default/files/2020-03/COVID-19\% 20-\%2013\%20March\%202020.pdf [Accessed 6 Jul 2021].

23 Khunti K, Kosiborod M, Ray KK. Legacy benefits of blood glucose, blood pressure and lipid control in individuals with diabetes and cardiovascular disease: time to overcome multifactorial therapeutic inertia? Diabetes Obes Metab 2018;20:1337-41.

24 Maddaloni E, Coraggio L, Pieralice S, et al. Effects of COVID-19 Lockdown on glucose control: continuous glucose monitoring data from people with diabetes on intensive insulin therapy. Diabetes Care 2020;43:e86-7.

25 Capaldo B, Annuzzi G, Creanza A, et al. Blood glucose control during Lockdown for COVID-19: CGM metrics in Italian adults with type 1 diabetes. Diabetes Care 2020;43:e88-9.

26 Cusinato M, Martino M, Sartori A, et al. Anxiety, depression, and glycemic control during Covid-19 pandemic in youths with type 1 diabetes. J Pediatr Endocrinol Metab 2021. doi:10.1515/jpem-2021-0153. [Epub ahead of print: 28 Jun 2021].

27 Robinson E, Boyland E, Chisholm A, et al. Obesity, eating behavior and physical activity during COVID-19 lockdown: a study of UK adults. Appetite 2021;156:104853. 
28 Robinson E, Gillespie S, Jones A. Weight-related lifestyle behaviours and the COVID-19 crisis: an online survey study of UK adults during social lockdown. Obes Sci Pract 2020;6:735-40.

29 Karatas S, Yesim T, Beysel S. Impact of lockdown COVID-19 on metabolic control in type 2 diabetes mellitus and healthy people. Prim Care Diabetes 2021;15:424-7.

30 Biamonte E, Pegoraro F, Carrone F, et al. Weight change and glycemic control in type 2 diabetes patients during COVID-19 pandemic: the lockdown effect. Endocrine 2021;72:604-10.

31 Tanji Y, Sawada S, Watanabe T, et al. Impact of COVID-19 pandemic on glycemic control among outpatients with type 2 diabetes in Japan: a hospital-based survey from a country without lockdown. Diabetes Res Clin Pract 2021;176:108840.

32 Rastogi A, Hiteshi P, Bhansali A. Improved glycemic control amongst people with long-standing diabetes during COVID-19 lockdown: a prospective, observational, nested cohort study. Int J Diabetes Dev Ctries 2020:476-81.

33 Ruissen MM, Regeer H, Landstra CP, et al. Increased stress, weight gain and less exercise in relation to glycemic control in people with type 1 and type 2 diabetes during the COVID-19 pandemic. BMJ Open Diabetes Res Care 2021;9.

34 Kanc K, Komel J, Kos M, et al. H(ome)bA1c testing and telemedicine: High satisfaction of people with diabetes for diabetes management during COVID-19 lockdown. Diabetes Res Clin Pract 2020;166:108285.

35 Wake DJ, Gibb FW, Kar P, et al. Endocrinology in the time of COVID-19: remodelling diabetes services and emerging innovation. Eur J Endocrinol 2020;183:G67-77.

36 Greenhalgh T, Wherton J, Shaw S, et al. Video consultations for covid-19. BMJ 2020;368:m998.

37 Phillip M, Bergenstal RM, Close KL, et al. The Digital/ Virtual diabetes clinic: the future is Now-Recommendations from an international panel on diabetes digital technologies introduction. Diabetes Technol Ther 2021;23:146-54.

38 Williams R, Jenkins DA, Ashcroft DM, et al. Diagnosis of physical and mental health conditions in primary care during the COVID-19 pandemic: a retrospective cohort study. Lancet Public Health 2020;5:e543-50.

39 Carr MJ, Steeg S, Webb RT, et al. Effects of the COVID-19 pandemic on primary care-recorded mental illness and selfharm episodes in the UK: a population-based cohort study. Lancet Public Health 2021;6:e124-35.

40 Mansfield KE, Mathur R, Tazare J, et al. Indirect acute effects of the COVID-19 pandemic on physical and mental health in the UK: a population-based study. Lancet Digit Health 2021;3:e217-30. 